\title{
Barriers of HIV testing among mentally ill persons in mental healthcare settings
}

\author{
Arupkumar Chakrabartty, Pampa Basu, Aditi K. Sarkar, Kazi M. Ali \\ Independent Researcher
}

\begin{abstract}
Due to certain risky behaviors, persons with severe mental illnesses are potentially prone to develop human immunodeficiency virus (HIV) infection more than people in general. Therefore, it is often argued that mentally ill persons should be considered as high risk groups, and so, they should go for HIV testing. For combating dual illnesses of HIV and mental illness together, there are several barriers that appear. Studies have been conducted to explore risk factors of HIV infection and HIV prevalence among mentally ill persons, and also on the probability that a mentally ill person get HIV tested compared with general population. However, hardly ever, studies have looked into barriers of HIV testing among mentally ill persons in mental healthcare settings. On this background, we have reviewed literature to explore barriers in performing HIV testing of a mentally ill person in mental healthcare settings. An important areas were found to act as barriers that added stigma due to dual illness of mental health and HIV, gender, type of facility, and its preparedness. Another barrier is the validity of the informed consent of a mentally ill person, which is required for a test. There are several conflicting situations on how to deal with this dual illness. The study recommends further exploratory researches on the matter and developing standard HIV testing protocol in mental healthcare settings.
\end{abstract}

HIV AIDS Rev 2017; 16, 2: 65-69 DOI: https://doi.org/10.5114/hivar.2017.68832

Key words: barriers, mentally ill, healthcare.

\section{Introduction}

Mentally ill persons, especially those with severe mental illness (SMI) like schizophrenia and mania, are several times at higher risk of human immunodeficiency virus (HIV) infection than general people due to their type and status of mental illness, unsafe sexual practices, and substance use [1]. There are some psychiatric conditions, which may predispose certain individuals to human immunodeficiency virus (HIV) infection due to its influence on their behaviors [2]. Because of the benefits of HIV testing, it is important for individuals with SMI to have routine access to testing [3]. In the United States, the rate of HIV infection among citizens who live with SMI is significantly higher than the general

Address for correspondence: Dr Arupkumar Chakrabartty, Health Vision and Research, Dream Home Apartment Govind 301, 700030 37/4A, Northern Avenue, India, phone: +919051353396, e-mail: arup.publication@gmail.com

population [4]. HIV seroprevalence among people with severe mental illness has increased substantially in recent years [5]. Compared to control men and women, bipolar men or women had more partners in the last year and sex without condoms [6]. Adults with SMI have been disproportionately affected by the HIV/AIDS epidemic. In a risk screening study among psychiatric patients newly admitted over a oneyear period to a state psychiatric hospital in New York City, HIV seroprevalence was $0.6 \%$ among the low-risk patients, but $14.4 \%$ among the high-risk patients. Among them, 23\% of patients were engaged in risky behaviors including injecting drugs [7]. There are appropriate evidences from the experiences in India examining the link between HIV and mental health. In a general hospital setting in South of India,

Article history:

Received: 5.01.2017

Received in revised form: 16.04.2017

Accepted: 26.04.2017

Available online: 05.07.2017
International Journal of HIV-Related Problems

HIV \& AIDS

R e v i e w 
HIV infection rate was $1.03 \%$ compared to the national seroprevalence rate of $0.7 \%$ [8-12]. HIV prevalence of $1.7 \%$ has been reported among psychiatric inpatients [13]. The predominant risk behavior among psychiatric patients in India is unprotected heterosexual intercourse, which is the most common mode of disease transmission in the country [14-16]. If a mentally ill person is at risk of HIV infection with unknown HIV status, and if contacted sexually with a general person without mental illness, there is a high probability of spreading the infection [1]. Therefore, it is suggested for timely HIV counseling and testing of mentally ill persons based on their risks [17]. Elevated prevalence of HIV in populations with mental illness and their probability to be HIV tested, suggests mandatory HIV test among people with SMI like schizophrenia, bipolar disorder, and depression [18]. Adults with SMI are in need of interventions promoting routine HIV testing and risk-reduction counseling [19]. In combating dual illness of HIV and mental illness, certain issues have come up. Few of them are validity of informed consent given by a mentally ill person, stigma attached to dual illness, preparedness of facility, and skills of the service providers. To handle these issues, there are no definite guidelines in India as per law, which mental healthcare providers can refer while serving in their settings. This leads to huge variations in adherence and practices among providers based on their own ethical values and perceptions [20-22].

\section{Aim of the study}

There is lack of information about different barriers that influence mentally ill persons in accessing HIV testing services, and effect HIV testing practices among mental healthcare providers. Therefore, the current literature review aims to describe different barriers in HIV testing for mentally ill persons in mental healthcare settings.

\section{Brief description of existing knowledge on the barriers of HIV testing of mentally ill persons}

\section{Ethics and informed consent}

Strict rules and guidelines affect everyday practice in all settings, but may operate differently in mental healthcare on ethical ground [20-22]. Obtaining informed consent from mentally ill persons involves educating, disclosing advantages, and disadvantages of testing for HIV, along with listening, answering questions, and seeking permission to proceed through each step of counseling and testing. In order to give a consent, patient must be considered competent and understand the purpose, risks, harms, and benefits of being tested. Consent must be voluntary [23, 24]. Regarding informed consent of a patient as a barrier in counseling or testing, there is a dilemma among providers in their perceptions. Validity of consent does not become a barrier for HIV test- ing among most of the providers in public settings. Waiting to get a valid consent, till a violent patient becomes calm and judgmental through admission and treatment for arranging HIV testing, may be justified as a valid argument. However, it should be ensured that the inpatients are not going to be affected and patient's confidentiality is protected. Protection of wife or any other sexual partner from an infected person is debatable. Around $80 \%$ of subjects with schizophrenia and $96 \%$ of the HIV positive patients demonstrated adequate capacity to consent for hypothetical circumstances [25]. There is a lack of concern about ethics in dealing with such issues among providers. Efforts are made to assess the changing trend of informed consent process among mentally challenged people for doing HIV research and factors shaping these changes [26]. Great harm may result from careless breach of a patient's trust. Physicians are responsible for ensuring that their nursing and support staff respect the confidentiality of information obtained during testing for HIV and counseling. Majority feels that HIV test result of mentally sound person is much different with mentally challenged person. In mental healthcare settings, results are not disclosed. Patients are directed to Voluntary Counseling and Testing Center (VCTC) of adjacent medical colleges. Impact of having HIV positive result is huge and devastating. Cognitive changes of a patient knowing his or her HIV positive status are disbelief, non-acceptance, stigma, life style, anger, aggression, or suicidal ideation. For a mentally challenged person, in days after disclosing the test result, the cognitive and behavioral aspects are not predictable. This has made HIV test more conflicting, and thus, a huge barrier for testing $[17,23,24]$.

\section{Stigma}

Some of the key of culturally relevant areas needs an enquiry like the role of stigma and disclosure in psychiatric morbidity, the role of spirituality as a protective coping mechanism, access to and cost of healthcare [2]. However, only a limited literature has focused on stigma in the context of co-occurrence of psychiatric disorders and HIV infection. When one-sided stigma coming from HIV/AIDS is superadded to another stigma coming from mental health problems, things become more complicated. Due to fear of stigma, people are reluctant to seek counseling or treatment. Ultimate effect on stigma due to HIV and mental illness together are sometimes additive, sometimes subtractive, or having no relation. Depending upon patient's illness variety, type of substance use, highness, even caste and education, stigma may be perceived differently [17]. Mental illness and stigma related to AIDS are very common in people living with HIV (PLHIV). Stigma and discrimination from that among PLHIV may have adverse impacts on their mental health conditions. There is a need for community-based interventions to reduce stigma and discrimination in the general public to cope with this situation [27]. Day to day activities of normal life are disrupted out of stigma, one's sense of control, and self-esteem. A dual illness of HIV infection 
and mental health problems may be threatening one's identity $[28,29]$. The effect of social stigma may lead to rejection in the workforce, discriminatory housing practices, and unsympathetic health care workers [30]. It is reported by the psychiatric service providers that stigma is more with substance users than those who are psychiatrically ill. In a study in Kolkata, one provider said: "we deal substance user as a client, not as an addict. We use the term "use" not 'abuse'. Substance use is a disease. Reducing stigma out of substance use needs special care for counseling, (...) so we stress on enhancing self-esteem of the client to reduce perceived stigma". Stigma is different in an individual, family, and community level. Family members of an HIV positive person are stigmatized. One provider said: "when mental illness is mixed with HIV, think no one will marry a girl from that family. Community perceives the stigma due to the notion of poor morale of the individual or family". Opinion psychologist said: "stigma for inpatient is nil as we can maintain $100 \%$ confidentiality. It is problem for outpatient department. Outpatient will perform all tests e.g. thyroid and blood routine tests, whatever. They come back with reports other than HIV test result at hand. It's simply due to stigma". Whereas opinion of another psychiatrist is different: "in indoor environment, stigma is much more due to fear of isolation from others. Neighboring of patients may know my status". One counselor said: "information of a HIV positive status spreads like volatile substance in closed environment, and that is why stigma is much more in inpatient department than outpatient" [17]. It obviously stands due to provider's lack of concerns, with no available guidelines at hand as how to deal such issues, and because message transmits faster, affecting privacy of a patient. Stigma is perceived differently across cultures and need to be dealt differently through culturally appropriate prevention strategy. Stigma arising from HIV is superimposed to stigma arising from mental health, and then things become more complicated. Stigma may have powerful consequences like depression, lack of self-worth, and despair [17]. For fear of disclosure, people are reluctant to seek for counseling or treatment. The most common internal stigma included guilty feeling $(53.1 \%)$ and feeling ashamed (45.9\%). Most of the internal stigma variables are linked with levels of mental disorders [27].

\section{Facility and its preparedness}

In a study in Gujarat in India, widespread stigma and misinformation about depression among psychiatric clinicians were found. This calls for the need of sensitization of mental health and HIV care providers in health facilities [31]. Good confidentiality is maintained in majorities of the public mental healthcare settings; however, it is poorer than facilities of Non-Government Organizations (NGO). Findings suggest that in NGOs, providers counsel in a separate room. In public settings, patients are standing in a row. Maintaining higher confidentiality may be related with differences in provider's perceptions or reporting bias. Item queries like type of substance use, detoxification, harm reduction, needle syringe exchange, and enquiring personal histories are more frequent in NGOs compared to public settings. There was uniformity in the idea that space, privacy, or confidentiality is not up to the level for all public settings. Most of the clients never give feedback when they are advised for HIV testing. Few respondents from NGOs has told that prevention of the contact persons is preferable than mandatory testing. Mandatory testing is recommended in circumstances where a patient is in a high-risk group, for a person being raped, performed unsafe sex with high-risk partner, or being injured (with history of blood exchange through injury) by a person with known positive status. The majority agrees that HIV testing should be mandatory for high-risk groups like IDUs, highly promiscuous, manic, or dementia patients, and also their contacts persons of high-risk groups [17]. The mental health profession remains as untapped resource within the community and mental healthcare settings for addressing combating mental health and HIV/AIDS $[32,33]$. HIV/AIDS continue to present a major challenges for mental health professionals. Screening for risk factors may also suffer because professionals are reluctant to bring up HIV with psychiatric patients, often out of well-meaning concern for the patients [34,35]. Providers may overestimate the anxiety caused by taking a sexual history. They may feel that discussing sexual behavior can worsen psychiatric symptoms. They may assume patient's sexual histories to be unreliable and see little point in obtaining them. However, there is evidence that patients give reliable information, and many patients welcome the opportunity to discuss sexual issues [20]. A study in New York revealed that in psychiatry departments, $47 \%$ providers conducted HIV risk assessment, $6 \%$ reported that they provided all information to all patients. Commonest practice is to provide information only to high-risk group. Around 34\% of provided information to selected individuals were doctors and nurses [36]. In one study in Kolkata among psychiatric providers, around 73\% providers considered that patients had poor acceptance for HIV screening, 79\% reported poor insight of patients, and $50 \%$ considered dual illness as most common barriers in providing services. Around 25\% providers identified more expenses due to dual illness as a barrier. High patient-rush was also identified as another barrier. A few providers were not aware of the Voluntary Counseling and Testing for HIV screening. Lack of proper coordination among providers was identified as another barrier [17].

\section{Gender}

A study in South India indicates gender-based difference in HIV risk behaviors such as exchanging money for sex and especially for women in linkage to gender based discrimination, to have sex for money [37]. In the study in Kolkata, there was no statistical significance between provider's gender perception having role in provision of HIV care with confidentiality, and sex of providers [17]. A study in Canada showed that gender is a more important variable in sexual risk assessment than the specialty of a clinician. 
General practitioners avoided screening as they did not feel their patients having risk of HIV. They were more concerned about patient discomfort [38]. Good strategies for the care of mental illness and risk reduction cannot be without gender. The relationship of gender and health-seeking behavior is a powerful determinant of gender differences [39]. In a study conducted in Kolkata in 2005, it is found that providers are only adhered to ask such questions only if they consider a patient to be a high risk. Public health implication is that there may remain hidden high-risk sexual behaviors that are unexplored as on the part of patients, if not explored through routine screening process [17]. Inhibition was reported mostly among women to talk freely on sexual behaviors leading to HIV infection. One counselor said: "as a women counselor, sometimes I feel unsafe in sexual health counseling. A patient may become erotic and scratch me. Patient feels that, I am dying, let others also die. Where is my safety?" [17]. It is important that in psychiatric treatment, the providers are addressing gender issues in both patients and professionals. However, the preparedness is scarce across all mental healthcare settings [40].

\section{Conclusions}

Important barriers found to act against HIV testing among mentally ill persons are ethical conflicts, including validity of the informed consent, stigma, gender, and type of facility. Whether HIV testing could be done for mentally ill person is well evidenced and discussed, compared to least discussion on barriers of dealing with the dual illness of mental health and HIV together in mental healthcare settings. In India under the National AIDS Control Program, there are no such guidelines helping mental healthcare providers in this matter. More researches would be required to explore the barriers, particularly the way they influence and the extent on adherence to HIV risk screening among mentally ill persons. Then decision makers may be interested to formulate policy under the National AIDS Control Program in India, or integrate the risk screening services in regular mental healthcare settings.

\section{Conflict of interest}

The author's declared no potential conflicts of interest with respect to the research, authorship, and/or publication of this article.

\section{References}

1. Walkup J, Satriano J, Barry D, et al. HIV Testing Policy and Serious Mental Illness. Am J Public Health 2002; 92: 1931-1939.

2. Chandra PS, Desai G, Ranjan S. HIV \& psychiatric disorders. Indian J Med Res 2005; 121: 451-467.

3. Senn TE, Carey MP. HIV testing among individuals with a severe mental illness: review, suggestions for research, and clinical implications. Psychol Med 2009; 39: 355-363.

4. Dyer JG, McGuinness TM. Reducing HIV risk among people with serious mental illness. J Psychosoc Nurs Ment Health Serv 2008; 46: 26-34.
5. Otto-Salaj LL, Stevenson LY. Influence of psychiatric diagnoses and symptoms on HIV risk behavior in adults with serious mental illness. AIDS Read 2001; 11: 197-208.

6. Downey J, Friedman RC, Haase E, et al. Comparison of Sexual Experience and Behavior between Bipolar Outpatients and Outpatients without Mood Disorders. Psychiatry J 2016; 216: 583-589.

7. Volavka J, Convit A, O'Donnell J, et al. Assessment of risk behaviors for HIV infection among psychiatric inpatients. Hosp Community Psychiatry 1992; 43: 482-485.

8. Grassi L, Pavanati M, Cardelli R, et al. HIV-risk behavior and knowledge about HIV/AIDS among patients with schizophrenia. Psychol Med 1999; 29: 171-179.

9. Collins PY, Holman AR, Freeman MC, et al. What is the relevance of mental health to HIV/AIDS care and treatment programs in developing countries? A systematic review. AIDS 2006; 20: 1571-1582.

10. Chandra PS, Ravi V, Puttaram S, et al. HIV and mental illness. Br J Psychiatry 1996; 168: 654.

11. Chopra MP, Eranti SS, Chandra PS. HIV-related risk behaviors among psychiatric inpatients in India. Psychiatr Serv 1998; 49: 823-825.

12. Tharyan P, Ramalingam S, Kannangai R, et al. Prevalence of HIV infection in psychiatric patients attending a general hospital in Tamil Nadu, South India. AIDS Care 2003; 15: 197-205.

13. Carey MP, Ravi V, Chandra PS, et al. Prevalence of HIV, Hepatitis B, syphilis, and chlamydia among adults seeking treatment for a mental disorder in southern India. AIDS Behav 2007; 11: 289-297.

14. Chandra PS, Carey MP, Carey KB, et al. HIV risk behavior among psychiatric inpatients: Results from a hospital-wide screening study in southern India. Int J STD AIDS 2003; 14: 532-538.

15. Chandra PS, Carey MP, Carey KB, et al. Sexual coercion and abuse among women with a severe mental illness in India: An exploratory investigation. Compr Psychiatry 2003; 44: 205-212.

16. Chandra PS, Deepthivarma S, Jairam KR, et al. Relationship of psychological morbidity and quality of life to illness-related disclosure among HIV-infected persons. J Psychosom Res 2003; 54: 199-203.

17. Arupkumar C. HIV Counselling, Testing and Referral Services in Kolkata - a Provider Perspective. St. Joseph's Press for Achutha Menon Centre for Health Science Studies 2005; 9: 3-35.

18. Baligh R, Cui W, Wiam W, et al. HIV testing among adults with mental illness in the United States. AIDS Patient Care STDS 2014; 28: 628-634.

19. Meade CS, Sikkema KJ. HIV risk behavior among adults with severe mental illness: a systematic review. Clin Psychol Rev 2005; 25: 433-457.

20. Kaplan M, Herman R, Courns F, et al. HIV Prevention for People with Mental Illness, A Training Manual for Mental Health Professionals. 6th ed. Columbia 2003; 2-12.

21. Perkins DV, Hudson BL, Gray DM, et al. Decisions and justifications by community mental health providers about hypothetical ethical dilemmas. Psychiatr Serv 1998; 49: 1317-1322.

22. Kovnick JA, Appelbaum PS, Hoge SK, et al. Competence to consent to research among long-stay inpatients with chronic schizophrenia. Psychiatr Serv 2003; 54: 1247-1252.

23. Centers for Disease Control and Prevention. Revised guidelines for HIV counseling, testing, and referral. MMWR Recomm Rep 2001; 50 (RR-19): 1-58.

24. NACO (National AIDS Control Organization), NACP (National AIDS Control Program), NACO Guidelines, VCTC Guidelines. Available from: www. naco.nic.in (Accessed: 21 April 2004).

25. Moser DJ, Schultz KS, Arndt S, et al. Capacity to provide informed consent for participation in schizophrenia and HIV research. Am J Psychiatry 2002; 159: 1201-1207.

26. McKay M. Informed Consent in AIDS and Mental Health Research. Global Institute of Public Health 2015; 2940: 5-13.

27. Yi S, Chhoun P, Suong S, et al. AIDS-related stigma and mental disorders among people living with HIV: a cross-sectional study in Cambodia. PLoS One 2015; 10: e0121461. 
28. Collins E, Wagner C, Walmsley S. Psychosocial impact of the lipodystrophy syndrome in HIV infection. AIDS Read 2000; 10: 546-550.

29. Link BG, Struening EL, Neese-Todd S, et al. Stigma as a barrier to recovery: The consequences of stigma for the self-esteem of people with mental illnesses. Psychiatr Serv 2001; 52: 1621-1626.

30. Parker R, Aggleton P. HIV and AIDS-related stigma and discrimination: a conceptual framework and implications for action. Soc Sci Med 2003; 57: 13-24.

31. Almanzar S, Shah N, Vithalani S, et al. Knowledge of and attitudes toward clinical depression among health providers in Gujarat, India. Ann Glob Health 2014; 80: 89-95.

32. Joore IK, Geerlings SE, Brinkman K, et al. The importance of registration of sexual orientation and recognition of indicator conditions for an adequate HIV risk-assessment. BMC Infect Dis 2017; 17: 178 .

33. Wolf MS, Mitchell CG. Preparing social workers to address HIV/ AIDS prevention and detection: implication for professional training and education. J Community Health 2002; 27: 165-179.

34. Wolf MS, Linsk NL, Mitchell CG, et al. HIV prevention in practice: an assessment of the public health response of physicians and nurses in Midwest. J Community Health 2004; 29: 63-73.

35. Walkup J, Satriano J, Hansell S, et al. Practices Related to HIV Risk Assessment in General Hospital Psychiatric Units in new York States. Psychiatric Serv 1998; 49: 529-530.

36. Walkup J, McAlpine DD, Olfson M, et al. Recent HIV Testing Among General Hospital Inpatients with Schizophrenia: Findings from Four New York City Sites. Psychiatric Quarterly 2000; 71: 177-193.

37. Chandra PS, Carey MP, Carey KB, et al. HIV risk behavior among psychiatric inpatients: results from a hospital-wide screening study in southern India. Int J STD AIDS 2003; 14: 532-538.

38. Haley N, Maheux B, Rivard M, et al. Sexual Health Risk Assessment and Counseling in Primary Care: How Involved Are General Practitioners and Obstetrician-Gynecologist? Am J Public Health 1999 89: 899-902.

39. Afifi M. Gender differences in mental health. Singapore Med J 2007; 48: 385-391.

40. Dora K. Gender and mental health: recognition of unresolved issues. Advances in Psychiatric Treatment 2001; 7: 83-84. 